QUARTERLY OF APPLIED MATHEMATICS

VOLUME LXIX, NUMBER 1

MARCH 2011, PAGES 91-103

S 0033-569X(2010)01200-7

Article electronically published on December 9, 2010

\title{
FLOCKING AND SYNCHRONIZATION OF PARTICLE MODELS
}

\author{
BY \\ SEUNG-YEAL HA (Department of Mathematical Sciences, Seoul National University, Seoul \\ 151-747, Korea), \\ CORRADO LATTANZIO (Department of Pure and Applied Mathematics, University of L'Aquila, \\ loc. Coppito, 67010 L'Aquila, Italy), \\ BRUNO RUBINO (Department of Pure and Applied Mathematics, University of L'Aquila, loc. \\ Coppito, 67010 L'Aquila, Italy), \\ AND \\ MARSHALL SLEMROD (Department of Mathematics, University of Wisconsin-Madison, Wisconsin \\ 53706-1388)
}

Abstract. In this note, we present a multi-dimensional flocking model rigorously derived from a vector oscillatory chain model and study the connection between the Cucker-Smale flocking model and the Kuramoto synchronization model appearing in the statistical mechanics of nonlinear oscillators. We provide an alternative direct approach for frequency synchronization to the Kuramoto model as an application of the flocking estimate for the Cucker-Smale model.

1. Introduction. The purpose of this paper is to unify some popular notions in the flocking and synchronization of dynamical systems. In particular, we first consider a theme already addressed by Ha and Slemrod [9]: the relation of two, usually separately studied, flocking models, i.e. the Cucker-Smale system [4, 5, 12] and the oscillator chain system [6] pioneered by Erdmann et al. Both systems are known to exhibit flocking behavior, but until the paper 9] the two models had not been linked. In the original 9] paper only a simple scalar version was presented, so we use this paper to present the general vector case where the flock propagates in $d$-dimensional Euclidean space. Of

Received July 17, 2009.

2000 Mathematics Subject Classification. Primary 92D25, 74A25, 76N10.

Key words and phrases. Flocking, particles, mechanical model, synchronization.

E-mail address: syha@snu.ac.kr

WWW address: http://www.math.snu.ac.kr/〜syha

E-mail address: corrado.lattanzio@univaq.it

WWW address: http://univaq.it/ corrado

E-mail address: bruno.rubino@univaq.it

$W W W$ address: http://univaq.it/〜rubino

E-mail address: slemrod@math.wisc.edu 
course, the value of such an approach is that it both unifies the theories and provides a rigorous proof of the flocking phenomena for both Cucker-Smale and the oscillator chain. As a second application of the flocking estimates, we show that the well-known Kuramoto synchronization model [10] also falls within our theory and hence a rigorous "flocking" theorem is immediately derivable.

The paper is divided into two sections after this Introduction. Section 2 is devoted to the oscillator chain and the application of Tikhonov's theorem to recover the CuckerSmale system. Flocking is then proved for the Cucker-Smale system. Section 3 recalls Kuramoto's synchronization model [10] and again derives a rigorous proof of flocking.

2. From mechanical model to flocking model. In this section, we present a new particle model which reveals the flocking phenomena and can be rigorously derived from a singularly perturbed mechanical system. This is a multi-dimensional analogue of the attempt given in $[9]$.

2.1. Formal derivation. Consider a simple mechanical model of mobile agents with a steep attracting force and a friction coefficient $(\varepsilon>0)$ moving in physical space $\mathbb{R}^{d}$ :

$$
\begin{aligned}
\frac{d x_{i}}{d t} & =v_{i} \\
\varepsilon \frac{d v_{i}}{d t} & =\frac{\lambda}{N} \sum_{j=1}^{N} \nabla_{x} \varphi\left(x_{j}-x_{i}\right)-v_{i}
\end{aligned}
$$

where $\lambda$ is the nonnegative coupling constant, and the mobile agents are represented by point particles with the same mass $\varepsilon$, which will set the time scale for the flocking to occur. Damping is needed as a self-organizing mechanism to remove energy from the configuration, a role which may be played by bird wings in flight. The force potential $\varphi$ is assumed to be isotropic and only dependent on the distance between two mobile agents: with abuse of notation, we set

$$
\varphi(x):=\varphi(|x|)
$$

Then the equation (2.1b) can be written as

$$
\varepsilon \frac{d v_{i}}{d t}=\frac{\lambda}{N} \sum_{j=1}^{N} \varphi^{\prime}\left(\left|x_{j}-x_{i}\right|\right) \frac{x_{j}-x_{i}}{\left|x_{j}-x_{i}\right|}-v_{i}
$$

We now take a time derivative of (2.2) to find the singularly perturbed second-order ODE system:

$$
\begin{aligned}
\varepsilon \frac{d^{2} v_{i}}{d t^{2}}= & \frac{\lambda}{N} \sum_{j=1}^{N}\left[\frac{\varphi^{\prime}\left(r_{i j}\right)}{r_{i j}}\left(v_{j}-v_{i}\right)+\left(\frac{\varphi^{\prime \prime}\left(r_{i j}\right)}{r_{i j}^{2}}-\frac{\varphi^{\prime}\left(r_{i j}\right)}{r_{i j}^{3}}\right)\left(\left(x_{j}-x_{i}\right) \cdot\left(v_{j}-v_{i}\right)\right)\left(x_{j}-x_{i}\right)\right] \\
& -\frac{d v_{i}}{d t}
\end{aligned}
$$


where $r_{i j}=\left|x_{j}-x_{i}\right|$. In the formal zero mass limit $\varepsilon \rightarrow 0$, the combined systems (2.1a) and (2.3) become

$$
\begin{aligned}
\frac{d x_{i}}{d t} & =v_{i}, \\
\frac{d v_{i}}{d t} & =\frac{\lambda}{N} \sum_{j=1}^{N}\left[\frac{\varphi^{\prime}\left(r_{i j}\right)}{r_{i j}}\left(v_{j}-v_{i}\right)+\left(\frac{\varphi^{\prime \prime}\left(r_{i j}\right)}{r_{i j}^{2}}-\frac{\varphi^{\prime}\left(r_{i j}\right)}{r_{i j}^{3}}\right)\left(\left(x_{j}-x_{i}\right) \cdot\left(v_{j}-v_{i}\right)\right)\left(x_{j}-x_{i}\right)\right] \\
& :=\frac{\lambda}{N} \sum_{j=1}^{N}\left[\psi_{1}\left(r_{i j}\right)\left(v_{j}-v_{i}\right)+\psi_{2}\left(r_{i j}\right)\left(\left(x_{j}-x_{i}\right) \cdot\left(v_{j}-v_{i}\right)\right)\left(x_{j}-x_{i}\right)\right] .
\end{aligned}
$$

Note that for a simple choice of $\varphi(x)=\frac{|x|^{2}}{2}$ (which corresponds to the potential in the harmonic oscillator problem with a unit spring constant), the coefficients in the second equation of (2.4) become

$$
\psi_{1}=1, \quad \psi_{2}=0
$$

hence we can recover the Cucker-Smale system with all-to-all communication rate. The new component in the system (2.4),

$$
\psi_{2}\left(r_{i j}\right)\left(\left(x_{j}-x_{i}\right) \cdot\left(v_{j}-v_{i}\right)\right)\left(x_{j}-x_{i}\right),
$$

seems to be related with the rotational motion of the flocking group. On the other hand, in the one-dimensional case $d=1$, the two terms in the R.H.S. of the second equation of (2.4) can be combined to result in the corresponding term in the Cucker-Smale system, i.e.,

$$
\begin{gathered}
\frac{\lambda}{N} \sum_{j=1}^{N}\left[\psi_{1}\left(r_{i j}\right)\left(v_{j}-v_{i}\right)+\psi_{2}\left(r_{i j}\right)\left(\left(x_{j}-x_{i}\right) \cdot\left(v_{j}-v_{i}\right)\right)\left(x_{j}-x_{i}\right)\right] \\
=\frac{\lambda}{N} \sum_{j=1}^{N}\left(\psi_{1}\left(r_{i j}\right)+\psi_{2}\left(r_{i j}\right) r_{i j}^{2}\right)\left(v_{j}-v_{i}\right) .
\end{gathered}
$$

This is why we do not see this new component in Ha and Slemrod's one-dimensional analysis in [9].

We next look for an admissible class of potentials $\varphi$ which guarantees the nonnegativity of the coefficients $\psi_{1}$ and $\psi_{2}$, i.e.,

$$
\psi_{1}(r)=\frac{\varphi^{\prime}(r)}{r} \geq 0, \quad \psi_{2}(r)=\frac{1}{r}\left(\frac{\varphi^{\prime}(r)}{r}\right)^{\prime} \geq 0 .
$$

By the direct observation of (2.5), we classify a potential $\varphi$ :

$$
\varphi(r):=\int_{0}^{r} s \psi_{1}(s) d s
$$

where $\psi_{1}$ satisfies

$$
\begin{aligned}
& \text { (i) } \psi_{1}>0, \quad \psi_{1}^{\prime}>0, \\
& \text { (ii) } \lim _{r \rightarrow 0^{+}} \psi_{1}(r)=R_{1}>0 .
\end{aligned}
$$


The second condition (ii) in (2.6) is imposed for the strict dissipativity of the total energy (see Lemma 2.1 and Theorem 2.3). Note that once $\psi_{1}$ is determined in the above process, then the communication rate $\psi_{2}$ is determined by the relation

$$
\psi_{2}(r)=\frac{1}{r} \psi_{1}^{\prime}(r) \text {. }
$$

On the other hand, the admissible conditions (2.6) can be expressed in terms of the potential $\varphi$ :

$$
\varphi^{\prime}>0, \quad \varphi^{\prime \prime} \geq \frac{\varphi^{\prime}}{r}, \quad \frac{\varphi^{\prime}}{r} \rightarrow R_{1} \quad \text { as } r \rightarrow 0^{+} .
$$

It is easy to see that the conditions (2.6) and (2.7) are equivalent to each other. Below, we present two admissible cases:

Examples. (i) Consider

$$
\psi_{1}(r)=e^{-\frac{1}{1+r}} .
$$

Then it is easy to see that this special choice satisfies the condition (2.6).

(ii) Consider

$$
\varphi(r)=r^{2}+r^{4} .
$$

Again, this potential satisfies (2.7).

2.2. Rigorous justification. In this section, we provide a rigorous justification for the passage from the singularly perturbed system (2.1a) and (2.3) to the system (2.4) as a direct application of Tikhonov's theorem.

We first rewrite the particle system (2.1a) and (2.3) as a first-order system:

$$
\begin{aligned}
\frac{d x_{i}}{d t} & =v_{i}, \quad 1 \leq i \leq N \\
\frac{d v_{i}}{d t} & =z_{i} \\
\varepsilon \frac{d z_{i}}{d t} & =\frac{\lambda}{N} \sum_{j=1}^{N}\left(\psi_{1}\left(r_{i j}\right)\left(v_{j}-v_{i}\right)+\psi_{2}\left(r_{i j}\right)\left(\left(x_{j}-x_{i}\right) \cdot\left(v_{j}-v_{i}\right)\right)\left(x_{j}-x_{i}\right)\right)-z_{i} .
\end{aligned}
$$

For the reader's convenience, we recall the classical theorem on the singular perturbation limit due to A. N. Tikhonov.

Consider the slow-fast dynamical system:

$$
\begin{aligned}
\frac{d y_{i}}{d t} & =f_{i}(y, z, t), \quad i=1, \ldots, n, \\
\mu_{j} \frac{d z_{j}}{d t} & =F_{j}(y, z, t), \quad j=1, \ldots, m,
\end{aligned}
$$

where $y=\left(y_{1}, \ldots, y_{n}\right), z=\left(z_{1}, \ldots, z_{m}\right)$, and $\mu_{j}$ are small positive parameters depending on a parameter $\mu$ in such a way that

$$
\lim _{\mu \rightarrow 0} \mu_{j}(\mu)=0, \quad \lim _{\mu \rightarrow 0} \frac{\mu_{j+1}}{\mu_{j}}=0 \quad \text { or } \quad 1 .
$$

Theorem 2.1 (Tikhonov [13]). Suppose the following conditions hold. 
(1) The degenerate systems obtained by setting all $\mu_{j}=0$,

$$
\frac{d y_{i}}{d t}=f_{i}(y, z, t), \quad F_{j}(y, z, t)=0
$$

have continuous solutions.

(2) The roots $z_{j}=\psi_{j}(y, t)$ of $F_{j}(y, z, t)=0$ have continuous first partial derivatives and are stable; i.e., the expression $\sum_{j}\left(z_{j}-\psi_{j}(y, t)\right) F_{j}(y, z, t)$ is negative in a suitable deleted neighborhood $\mathcal{N}$ of the roots.

Then as $\mu \rightarrow 0$, the solutions of (2.9) tend to the corresponding solutions of the degenerate system with the initial data $\left(y_{i}^{0}, z_{j}^{0}, t^{0}\right) \in \mathcal{N}$, and this convergence is uniform in a closed interval $\left[t_{1}, T_{*}\right]$ for any $t_{1}>t^{0}$ and a constant $T_{*}>t_{1}$.

In our case, $F_{j}$ is the right-hand side of (2.8c). The roots of $F_{j}=0$ are of course the sum on the right-hand side of (2.8c), and hence the second assertion (2) in Tikhonov's theorem above becomes

$$
\begin{aligned}
& \sum_{i=1}^{N}\left(z_{i}-\frac{\lambda}{N} \sum_{j=1}^{N}\left(\psi_{1}(r)\left(v_{j}-v_{i}\right)+\psi_{2}(r)\left(\left(x_{j}-x_{i}\right) \cdot\left(v_{j}-v_{i}\right)\right)\left(x_{j}-x_{i}\right)\right)\right) \\
& \cdot\left(\frac{\lambda}{N} \sum_{j=1}^{N}\left(\psi_{1}(r)\left(v_{j}-v_{i}\right)+\psi_{2}(r)\left(\left(x_{j}-x_{i}\right) \cdot\left(v_{j}-v_{i}\right)\right)\left(x_{j}-x_{i}\right)\right)-z_{i}\right)<0,
\end{aligned}
$$

which is trivially true in the whole state space, excluding the roots themselves.

TheOREm 2.2. Let $\left(x_{i}^{\varepsilon}(t), v_{i}^{\varepsilon}(t), z_{i}^{\varepsilon}(t)\right)$ be the solution to the singularly perturbed system (2.8a) - (2.8c) with initial data $\left(x_{i}(0), v_{i}(0), z_{i}(0)\right)$. Then for any small positive time $t_{1}$ and a given $T_{*}>t_{1}$, the solutions $\left(x_{i}^{\varepsilon}(t), v_{i}^{\varepsilon}(t), z_{i}^{\varepsilon}(t)\right)$ converge to $\left(x_{i}^{0}(t), v_{i}^{0}(t), z_{i}^{0}(t)\right)$ uniformly in $t \in\left[t_{1}, T_{*}\right]$, where $\left(x_{i}^{0}(t), v_{i}^{0}(t), z_{i}^{0}(t)\right)$ satisfy the unperturbed system:

$$
\begin{aligned}
\frac{d x_{i}^{0}}{d t} & =v_{i}^{0}, \quad t \geq 0, \quad 1 \leq i \leq N, \\
\frac{d v_{i}^{0}}{d t} & =z_{i}^{0}, \\
z_{i}^{0} & =\frac{\lambda}{N} \sum_{j=1}^{N}\left(\psi_{1}\left(r^{0}\right)\left(v_{j}^{0}-v_{i}^{0}\right)+\psi_{2}\left(r^{0}\right)\left[\left(x_{j}^{0}-x_{i}^{0}\right) \cdot\left(v_{j}^{0}-v_{i}^{0}\right)\right]\left(x_{j}^{0}-x_{i}^{0}\right)\right) .
\end{aligned}
$$

Proof. The proof follows from a direct application of Theorem 2.1. A more modern approach which generalizes Tikhonov's original idea as well as the method of averaging is in Artstein and Vigodner [2, and we also refer to [14 for reference. 
2.3. Flocking estimate. In this part, we study the flocking estimate for the formally derived new system from the mechanical particle system:

$$
\begin{aligned}
\frac{d x_{i}}{d t}= & v_{i} \\
\frac{d v_{i}}{d t}= & \frac{\lambda}{N} \sum_{j=1}^{N} \psi_{1}\left(\left|x_{j}-x_{i}\right|\right)\left(v_{j}-v_{i}\right) \\
& +\frac{\lambda}{N} \sum_{j=1}^{N} \psi_{2}\left(\left|x_{j}-x_{i}\right|\right)\left(\left(x_{j}-x_{i}\right) \cdot\left(v_{j}-v_{i}\right)\right)\left(x_{j}-x_{i}\right) .
\end{aligned}
$$

Lemma 2.1. Let $\left(x_{i}, v_{i}\right)$ be the solutions to the system 2.11a 2.11b. Then the total momentum and energy satisfy

$$
\begin{aligned}
\text { (i) } \frac{d}{d t} \sum_{i=1}^{N} v_{i}=0, & \\
\text { (ii) } \frac{d}{d t} \sum_{i=1}^{N}\left|v_{i}\right|^{2}=- & \frac{\lambda}{N} \sum_{1 \leq i, j \leq N} \psi_{1}\left(\left|x_{j}-x_{i}\right|\right)\left|v_{j}-v_{i}\right|^{2} \\
& \quad-\frac{\lambda}{N} \sum_{1 \leq i, j \leq N} \psi_{2}\left(\left|x_{j}-x_{i}\right|\right)\left|\left(x_{j}-x_{i}\right) \cdot\left(v_{j}-v_{i}\right)\right|^{2} .
\end{aligned}
$$

Proof. (i) The conservation of momentum is due to the fact that the right-hand side of (2.11b) is skew-symmetric under the transformation $i \leftrightarrow j$.

(ii) For the energy dissipation estimate, we take an inner product with (2.11b) and $2 v_{i}$, and then sum it over $i$ to find the desired result.

REMARK 2.1. Compared to the dissipation estimate for the Cucker-Smale flocking system [4, 5, 7, 8, we have an extra dissipation term

$$
\frac{\lambda}{N} \sum_{1 \leq i, j \leq N} \psi_{2}\left(\left|x_{j}-x_{i}\right|\right)\left|\left(x_{j}-x_{i}\right) \cdot\left(v_{j}-v_{i}\right)\right|^{2},
$$

which enhances the flocking.

For the flocking estimate, we first introduce averaged quantities and fluctuations around them:

$$
\langle x\rangle:=\frac{1}{N} \sum_{i=1}^{N} x_{i}, \quad\langle v\rangle:=\frac{1}{N} \sum_{i=1}^{N} v_{i},
$$

and

$$
\bar{x}_{i}:=x_{i}-\langle x\rangle, \quad \bar{v}_{i}:=v_{i}-\langle v\rangle .
$$

Then it easy to see that the dynamics for the averaged quantities and fluctuations are completely decoupled so that

$$
\langle v(t)\rangle=\langle v(0)\rangle, \quad\langle x(t)\rangle=\langle x(0)\rangle+t\langle v(0)\rangle,
$$


and

$$
\begin{aligned}
\frac{d \bar{x}_{i}}{d t}= & \bar{v}_{i} \\
\frac{d \bar{v}_{i}}{d t}= & \frac{\lambda}{N} \sum_{j=1}^{N} \psi_{1}\left(\left|\bar{x}_{j}-\bar{x}_{i}\right|\right)\left(\bar{v}_{j}, \bar{v}_{i}\right) \\
& +\frac{\lambda}{N} \sum_{j=1}^{N} \psi_{2}\left(\left|\bar{x}_{j}-\bar{x}_{i}\right|\right)\left(\left(\bar{x}_{j}-\bar{x}_{i}\right) \cdot\left(\bar{v}_{j}-\bar{v}_{i}\right)\right)\left(\bar{x}_{j}-\bar{x}_{i}\right) .
\end{aligned}
$$

For the flocking estimate, it suffices to get the dissipation estimate for the system (2.12a)(2.12b).

Theorem 2.3. The limit system (2.11a) (2.11b) admits flocking in the time-asymptotic limit. More precisely, for any solution $\left(x_{i}, v_{i}\right)$ with a finite initial energy for perturbation, we have

$$
\begin{aligned}
& \text { (i) }\left(\sum_{i=1}^{N}\left|\bar{v}_{i}(t)\right|^{2}\right) \leq e^{-2 \lambda R_{1} t}\left(\sum_{i=1}^{N}\left|\bar{v}_{i}(0)\right|^{2}\right), \quad t>0, \\
& \text { (ii) } \max _{1 \leq i \leq N}\left|\bar{x}_{i}(t)\right| \leq \max _{1 \leq i \leq N}\left|\bar{x}_{i 0}\right|+\frac{1}{\lambda R_{1}}\left(\sum_{i=1}^{N}\left|\bar{v}_{i}(0)\right|^{2}\right)^{\frac{1}{2}},
\end{aligned}
$$

where $R_{1}$ is a positive constant appearing in (2.6).

Proof. (i) As in Lemma 2.1] we have

$$
\begin{aligned}
\frac{d}{d t} \sum_{i=1}^{N}\left|\bar{v}_{i}\right|^{2}= & -\frac{\lambda}{N} \sum_{1 \leq i, j \leq N} \psi_{1}\left(\left|\bar{x}_{j}-\bar{x}_{i}\right|\right)\left|\bar{v}_{j}-\bar{v}_{i}\right|^{2} \\
& -\frac{\lambda}{N} \sum_{1 \leq i, j \leq N} \psi_{2}\left(\left|\bar{x}_{j}-\bar{x}_{i}\right|\right)\left|\left(\bar{x}_{j}-\bar{x}_{i}\right) \cdot\left(\bar{v}_{j}-\bar{v}_{i}\right)\right|^{2} \\
\leq & -\frac{\lambda}{N} \sum_{1 \leq i, j \leq N} \psi_{1}\left(\left|\bar{x}_{j}-\bar{x}_{i}\right|\right)\left|\bar{v}_{j}-\bar{v}_{i}\right|^{2} \\
\leq & -2 \lambda R_{1} \sum_{i=1}^{N}\left|\bar{v}_{i}\right|^{2} .
\end{aligned}
$$

We now integrate the above Gronwall's inequality to get the desired result.

(ii) It follows from the result in (i) that

$$
\left|\bar{v}_{i}(t)\right| \leq e^{-\lambda R_{1} t}\left(\sum_{i=1}^{N}\left|\bar{v}_{i}(0)\right|^{2}\right)^{\frac{1}{2}} .
$$

We now use the above estimate to find

$$
\left|\bar{x}_{i}(t)-\bar{x}_{i 0}\right| \leq \int_{0}^{t}\left|\bar{v}_{i}(s)\right| d s \leq \frac{1}{\lambda R_{1}}\left(\sum_{i=1}^{N}\left|\bar{v}_{i}(0)\right|^{2}\right)^{\frac{1}{2}}\left(1-e^{-\lambda R_{1} t}\right) .
$$

This yields the desired result. 
REMARK 2.2. For the flocking estimates for the particle and kinetic Cucker-Smale model, we refer to [3, 4, 5, 7, 8, 12,

As a corollary of Theorem 2.2 and Theorem 2.3 we obtain estimates for relative velocities and the flocking group's spatial diameter for the singularly perturbed system (2.8a)-(2.8c).

Corollary 2.1. Let $\varepsilon>0$ be sufficiently small. Then for any small positive time $t_{1}$ and a given $T_{*}>t_{1}$, there exists a positive constant $C$ such that the perturbed solutions $\left(x_{i}^{\varepsilon}, v_{i}^{\varepsilon}\right)$ to 2.8a - 2.8C) satisfy

$$
\begin{aligned}
& \text { (i) }\left|v_{i}^{\varepsilon}(t)-v_{j}^{\varepsilon}(t)\right|<2 \varepsilon+C e^{-\lambda R_{1} t}, \quad t \in\left[t_{1}, T_{*}\right], \\
& \text { (ii) }\left|x_{i}^{\varepsilon}(t)-x_{j}^{\varepsilon}(t)\right| \leq 2 \varepsilon+C .
\end{aligned}
$$

Here $\left(x_{i}^{0}(0), v_{i}^{0}(0)\right)$ is the initial datum corresponding to the unperturbed solution $\left(x_{i}^{0}(t), v_{i}^{0}(t)\right)$, and the constant $C$ depends only on the initial datum of the unperturbed solution.

Proof. It follows from Theorem 2.2 that $\exists t_{1}, T_{*}>0$ such that

$$
\max _{1 \leq i \leq N}\left|v_{i}^{\varepsilon}(t)-v_{i}^{0}(t)\right|<\varepsilon, \quad \max _{1 \leq i \leq N}\left|x_{i}^{\varepsilon}(t)-x_{i}^{0}(t)\right|<\varepsilon, \quad t \in\left[t_{1}, T_{*}\right] .
$$

On the other hand, we use Theorem 2.3 to estimate

$$
\max _{1 \leq i, j \leq N}\left|v_{i}^{0}(t)-v_{j}^{0}(t)\right| \leq\left(2 \sum_{i=1}^{N}\left|\bar{v}_{i}^{0}(t)\right|^{2}\right)^{\frac{1}{2}} \leq\left(2 \sum_{i=1}^{N}\left|\bar{v}_{i}^{0}(0)\right|^{2}\right)^{\frac{1}{2}} e^{-\lambda R_{1} t}, \quad t \in[0, \infty) .
$$

We simply apply the triangle inequalities:

$$
\begin{aligned}
\left|v_{i}^{\varepsilon}(t)-v_{j}^{\varepsilon}(t)\right| & \leq\left|v_{i}^{\varepsilon}(t)-v_{i}^{0}(t)\right|+\left|v_{j}^{\varepsilon}(t)-v_{j}^{0}(t)\right|+\left|v_{i}^{0}(t)-v_{j}^{0}(t)\right|, \quad t \in\left[t_{1}, T_{*}\right] \\
& \leq 2 \varepsilon+\left(2 \sum_{i=1}^{N}\left|\bar{v}_{i}^{0}(0)\right|^{2}\right)^{\frac{1}{2}} e^{-\lambda R_{1} t} .
\end{aligned}
$$

We use Theorem 2.3 to find

$$
\begin{aligned}
\left|x_{i}^{0}(t)-x_{j}^{0}(t)\right| & =\left|\bar{x}_{i}^{0}(t)-\bar{x}_{j}^{0}(t)\right| \\
& \leq\left|\bar{x}_{i}^{0}(0)-\bar{x}_{j}^{0}(0)\right|+\int_{0}^{t}\left|\bar{v}_{i}(s)-\bar{v}_{j}(s)\right| d s \\
& \leq\left|\bar{x}_{i}^{0}(0)-\bar{x}_{j}^{0}(0)\right|+\frac{1}{\lambda R_{1}}\left(2 \sum_{i=1}^{N}\left|\bar{v}_{i}^{0}(0)\right|^{2}\right)^{\frac{1}{2}} .
\end{aligned}
$$

Similarly, we have

$$
\begin{aligned}
\left|x_{i}^{\varepsilon}(t)-x_{j}^{\varepsilon}(t)\right| & \leq\left|x_{i}^{\varepsilon}(t)-x_{i}^{0}(t)\right|+\left|x_{j}^{\varepsilon}(t)-x_{j}^{0}(t)\right|+\left|x_{i}^{0}(t)-x_{j}^{0}(t)\right|, \quad t \in\left[t_{1}, T_{*}\right] \\
& \leq 2 \varepsilon+\left|\bar{x}_{i}^{0}(0)-\bar{x}_{j}^{0}(0)\right|+\frac{1}{\lambda R_{1}}\left(2 \sum_{i=1}^{N}\left|\bar{v}_{i}^{0}(0)\right|^{2}\right)^{\frac{1}{2}} .
\end{aligned}
$$


3. From the Kuramoto model to the Cucker-Smale model. In this section, we present a rather interesting bridge between the Kuramoto phase model and the CuckerSmale flocking model.

Consider the Kuramoto phase model describing the dynamics of nonlinear oscillators $\left\{x_{k}=e^{i \theta_{k}}\right\}$ moving on the circle with randomly distributed natural frequency $\Omega_{k}$ and sinusoidal coupling between oscillators:

$$
\frac{d \theta_{k}}{d t}=\Omega_{k}+\frac{\lambda}{N} \sum_{h=1}^{N} \sin \left(\theta_{h}-\theta_{k}\right), \quad t>0, \quad k=1, \ldots, N,
$$

subject to the initial phase

$$
\theta_{k}(0)=\theta_{k 0}, \quad k=1, \ldots, N .
$$

The Kuramoto model has been extensively studied in the last thirty years after Kuramoto's seminal work [10] and is still a popular subject for the modeling of synchronization phenomena arising from statistical physics (see a review article [1]). In particular, Kuramoto [10] observed that for the model in the $N \rightarrow \infty$ limit, there is a continuous dynamical phase transition at a critical value of the coupling strength $\lambda_{c}$. In particular, for $\lambda>\lambda_{c}$, the system shows the phase and frequency synchronization. We now differentiate the Kuramoto model to derive the dynamics of the frequency $\omega_{i}=\dot{\theta}_{i}$ :

$$
\frac{d \omega_{i}}{d t}=\frac{\lambda}{N} \sum_{j=1}^{N} \cos \left(\theta_{j}-\theta_{i}\right)\left(\omega_{j}-\omega_{i}\right), \quad t>0, \quad i=1, \ldots, N
$$

We take the initial frequency of oscillators to be the natural frequency of oscillators, i.e.,

$$
\omega_{i}(0)=\Omega_{i}, \quad i=1, \ldots, N .
$$

3.1. Dynamics of standard deviations. In this part, we present the dynamics of standard deviations for physical variables. We introduce averaged natural frequency, phase and frequency:

$$
\langle\Omega\rangle:=\frac{1}{N} \sum_{i=1}^{N} \Omega_{i}, \quad\langle\theta\rangle:=\frac{1}{N} \sum_{i=1}^{N} \theta_{i}, \quad\langle\omega\rangle:=\frac{1}{N} \sum_{i=1}^{N} \omega_{i} .
$$

Note that $\langle\Omega\rangle$ is a stationary random variable, and $\langle\theta\rangle,\langle\omega\rangle$ satisfy

$$
\langle\theta(t)\rangle=\left\langle\theta_{0}\right\rangle+t\langle\Omega\rangle, \quad\langle\omega(t)\rangle=\langle\Omega\rangle .
$$

We next introduce fluctuations of the state variables around the averaged quantities:

$$
\bar{\Omega}_{i}:=\Omega_{i}-\langle\Omega\rangle, \quad \bar{\theta}_{i}:=\theta_{i}-\langle\theta\rangle, \quad \bar{\omega}_{i}=\omega_{i}-\langle\omega\rangle .
$$

Then the fluctuations $\bar{\theta}_{i}$ and $\bar{\omega}_{i}$ satisfy

$$
\begin{aligned}
& \frac{d \bar{\theta}_{i}}{d t}=\bar{\Omega}_{i}+\frac{\lambda}{N} \sum_{j=1}^{N} \sin \left(\bar{\theta}_{j}-\bar{\theta}_{i}\right), \\
& \frac{d \bar{\omega}_{i}}{d t}=\frac{\lambda}{N} \sum_{j=1}^{N} \cos \left(\bar{\theta}_{j}-\bar{\theta}_{i}\right)\left(\bar{\omega}_{j}-\bar{\omega}_{i}\right) .
\end{aligned}
$$


Note that the equation (3.1b takes the form of the Cucker-Smale model in the regime $\cos \left(\theta_{j}-\theta_{i}\right)>0$. At this point, we denote with $\sigma_{\theta}, \sigma_{\omega}$ and $\sigma_{\Omega}$ the standard deviations of $\theta_{i}, \omega_{i}$ and $\Omega_{i}$ respectively:

$$
\sigma_{\theta}^{2}:=\frac{1}{N} \sum_{i=1}^{N}\left|\bar{\theta}_{i}\right|^{2}, \quad \sigma_{\omega}^{2}:=\frac{1}{N} \sum_{i=1}^{N}\left|\bar{\omega}_{i}\right|^{2}, \quad \sigma_{\Omega}^{2}:=\frac{1}{N} \sum_{i=1}^{N}\left|\bar{\Omega}_{i}\right|^{2} .
$$

We study the dynamics of these quantities in the following lemma.

Lemma 3.1. Let $\left(\bar{\theta}_{i}, \bar{\omega}_{i}\right)$ be smooth solutions to the system (3.1a) and (3.1b). Then the $\sigma_{\theta}$ and $\sigma_{\omega}$ standard deviations satisfy

$$
\begin{aligned}
& \text { (i) } \frac{d}{d t} \sum_{i=1}^{N}\left|\bar{\theta}_{i}\right|^{2}=2 \sum_{i} \bar{\theta}_{i} \bar{\Omega}_{i}-\frac{\lambda}{N} \sum_{1 \leq i, j \leq N}\left(\bar{\theta}_{j}-\bar{\theta}_{i}\right) \sin \left(\bar{\theta}_{j}-\bar{\theta}_{i}\right), \\
& \text { (ii) } \frac{d}{d t} \sum_{i=1}^{N}\left|\bar{\omega}_{i}\right|^{2}=-\frac{\lambda}{N} \sum_{1 \leq i, j \leq N} \cos \left(\bar{\theta}_{j}-\bar{\theta}_{i}\right)\left|\bar{\omega}_{j}-\bar{\omega}_{i}\right|^{2} .
\end{aligned}
$$

Proof. We only consider the second identity; the first identity can be treated similarly. We multiply the equation (3.1b) by $2 \bar{\omega}_{i}$, sum over $i$ and use the symmetry trick $i \leftrightarrow j$ to find

$$
\begin{aligned}
\frac{d}{d t} \sum_{i=1}^{N}\left|\bar{\omega}_{i}\right|^{2} & =\frac{2 \lambda}{N} \sum_{i, j} \cos \left(\left|\bar{\theta}_{j}-\bar{\theta}_{i}\right|\right) \bar{\omega}_{i} \cdot\left(\bar{\omega}_{j}-\bar{\omega}_{i}\right) \\
& =-\frac{2 \lambda}{N} \sum_{i, j} \cos \left(\left|\bar{\theta}_{j}-\bar{\theta}_{i}\right|\right) \bar{\omega}_{j} \cdot\left(\bar{\omega}_{j}-\bar{\omega}_{i}\right) \\
& =-\frac{\lambda}{N} \sum_{i, j} \cos \left(\left|\bar{\theta}_{j}-\bar{\theta}_{i}\right|\right)\left|\bar{\omega}_{j}-\bar{\omega}_{i}\right|^{2} .
\end{aligned}
$$

We next proceed with a dissipation estimate for the system (3.1a) and (3.1b). To this aim, we estimate the dynamics of the standard deviations in the following proposition.

Proposition 3.1. For given $T>0$, let $\left(\bar{\theta}_{i}, \bar{\omega}_{i}\right) \in C^{1}([0, T))$ be solutions to the system (3.1a) and (3.1b) satisfying the following stability condition:

$$
\sup _{0 \leq t<T} \sigma_{\theta}(t)<\frac{\pi}{4 \sqrt{N}}
$$

Then the standard deviations $\sigma_{\theta}$ and $\sigma_{\omega}$ satisfy the system of differential inequalities:

$$
\begin{aligned}
& \text { (i) }\left|\frac{d \sigma_{\theta}}{d t}\right| \leq \sigma_{\omega}, \\
& \text { (ii) } \frac{d \sigma_{\omega}}{d t} \leq-\lambda \cos \left(2 \sqrt{N} \sigma_{\theta}\right) \sigma_{\omega} .
\end{aligned}
$$

Proof. (i) We use the definitions of variances $\sigma_{\theta}^{2}, \sigma_{\omega}^{2}$ and $\frac{d \bar{\theta}_{i}}{d t}=\bar{\omega}_{i}$ to find

$$
\left|\frac{d \sigma_{\theta}^{2}}{d t}\right|=\frac{1}{N}\left|\frac{d}{d t} \sum_{i=1}^{N}\left(\bar{\theta}_{i}\right)^{2}\right| \leq \frac{2}{N} \sum_{i=1}^{N}\left|\bar{\theta}_{i}\right|\left|\bar{\omega}_{i}\right| \leq \frac{2}{N}\left(\sum_{i=1}^{N}\left|\bar{\theta}_{i}\right|^{2}\right)^{\frac{1}{2}}\left(\sum_{i=1}^{N}\left|\bar{\omega}_{i}\right|^{2}\right)^{\frac{1}{2}},
$$

where we used Cauchy-Schwarz's inequality. 
On the other hand, note that

$$
\left|\frac{d \sigma_{\theta}^{2}}{d t}\right|=2 \sigma_{\theta}\left|\frac{d \sigma_{\theta}}{d t}\right|, \quad \sum_{i=1}^{N}\left|\bar{\theta}_{i}\right|^{2}=N \sigma_{\theta}^{2}, \quad \sum_{i=1}^{N}\left|\bar{\omega}_{i}\right|^{2}=N \sigma_{\omega}^{2} .
$$

Thus in (3.3) we have

$$
2 \sigma_{\theta}\left|\frac{d \sigma_{\theta}}{d t}\right| \leq 2 \sigma_{\theta} \sigma_{\omega}
$$

and we divide the above inequality by $\sigma_{\theta}$ to find the desired result.

(ii) We use $\left|\bar{\theta}_{j}-\bar{\theta}_{i}\right| \leq 2 \sqrt{N} \sigma_{\theta} \leq \frac{\pi}{2}$ and the nonincreasing property of $\cos (\theta)$ in the interval $\left[0, \frac{\pi}{2}\right]$ to find

$$
\cos \left(\bar{\theta}_{j}-\bar{\theta}_{i}\right) \geq \cos \left(2 \sqrt{N} \sigma_{\theta}\right)
$$

On the other hand, note that

$$
\begin{aligned}
2 \sigma_{\omega} \frac{d \sigma_{\omega}}{d t} & =\frac{d \sigma_{\omega}^{2}}{d t}=\frac{1}{N} \frac{d}{d t} \sum_{i=1}^{N}\left|\bar{\omega}_{i}\right|^{2}=-\frac{\lambda}{N^{2}} \sum_{1 \leq i, j \leq N} \cos \left(\bar{\theta}_{j}-\bar{\theta}_{i}\right)\left|\bar{\omega}_{j}-\bar{\omega}_{i}\right|^{2} \\
& \leq-2 \lambda \cos \left(2 \sqrt{N} \sigma_{\theta}\right) \sigma_{\omega}^{2},
\end{aligned}
$$

where we used

$$
\sum_{1 \leq i, j \leq N}\left|\bar{\omega}_{i}-\bar{\omega}_{j}\right|^{2}=2 N \sum_{i=1}^{N}\left|\bar{\omega}_{i}\right|^{2}=2 N^{2} \sigma_{\omega}^{2} .
$$

3.2. Frequency synchronization. In this part, we study the dissipation estimate for standard deviations, which will give the existence of a frequency locked state:

$$
\omega_{i}=\omega_{j}, \quad i \neq j .
$$

The Lyapunov approach employed in this section was introduced in [7] in the context of the Cucker-Smale system with general communication rates. Consider the following system of differential inequalities:

$$
\left|\frac{d \sigma_{\theta}}{d t}\right| \leq \sigma_{\omega}, \quad \frac{d \sigma_{\omega}}{d t} \leq-\lambda \cos \left(2 \sqrt{N} \sigma_{\theta}\right) \sigma_{\omega}
$$

and define Lyapunov functionals

$$
\mathcal{H}^{ \pm}\left[\sigma_{\theta}(t), \sigma_{\omega}(t)\right]:=\sigma_{\omega}(t) \pm \lambda \int_{\sigma_{\theta}(0)}^{\sigma_{\theta}(t)} \cos (2 \sqrt{N} s) d s .
$$

Proposition 3.2 (Dissipation estimate). For given $T>0$, let $\left(\sigma_{\theta}, \sigma_{\omega}\right) \in C^{1}([0, T))$ be solutions to the system (3.1a) and (3.1b) satisfying the stability condition (3.2). Then the following stability estimate holds. For $t \geq 0$,

$$
\begin{aligned}
& \text { (i) } \mathcal{H}^{ \pm}\left[\sigma_{\theta}(t), \sigma_{\omega}(t)\right] \leq \mathcal{H}^{ \pm}\left[\sigma_{\theta}(0), \sigma_{\omega}(0)\right] \\
& \text { (ii) } \sigma_{\omega}(t)+\lambda\left|\int_{\sigma_{\theta}(0)}^{\sigma_{\theta}(t)} \cos (2 \sqrt{N} s) d s\right| \leq \sigma_{\omega}(0) .
\end{aligned}
$$


Proof. (i) We use Proposition 3.1, that is, relations (3.4), to show that

$$
\begin{aligned}
\frac{d}{d t} \mathcal{H}^{ \pm}[t] & =\frac{d}{d t} \sigma_{\omega}(t) \pm \lambda \cos \left(2 \sqrt{N} \sigma_{\theta}(t)\right) \frac{d}{d t} \sigma_{\theta}(t) \\
& \leq-\lambda \cos \left(2 \sqrt{N} \sigma_{\theta}\right) \sigma_{\omega} \pm \lambda \cos \left(2 \sqrt{N} \sigma_{\theta}(t)\right) \frac{d}{d t} \sigma_{\theta}(t) \\
& =\lambda \cos \left(2 \sqrt{N} \sigma_{\theta}\right)\left(-\sigma_{\omega} \pm \frac{d}{d t} \sigma_{\theta}(t)\right) \\
& \leq 0 .
\end{aligned}
$$

(ii) It follows from the result in (i) that we have

$$
\begin{gathered}
\sigma_{\omega}(t)+\lambda \int_{\sigma_{\theta}(0)}^{\sigma_{\theta}(t)} \cos (2 \sqrt{N} s) d s \leq \sigma_{\omega}(0), \\
\sigma_{\omega}(t)+\lambda \int_{\sigma_{\theta}(t)}^{\sigma_{\theta}(0)} \cos (2 \sqrt{N} s) d s \leq \sigma_{\omega}(0),
\end{gathered}
$$

which concludes the proof.

Finally we can prove our main result.

Theorem 3.1 (Frequency synchronization). Suppose the standard deviations of the initial configurations $\sigma_{\theta}(0)$ and $\sigma_{\omega}(0)$ satisfy

$$
\sigma_{\theta}(0)<\frac{\pi}{16 \sqrt{N}}, \quad \sigma_{\omega}(0)<\frac{\lambda \pi}{16 \sqrt{2 N}} .
$$

Then we have

$$
\begin{aligned}
& \text { (i) } \quad \sigma_{\omega}(t) \leq \sigma_{\omega}(0) e^{-\frac{\lambda t}{\sqrt{2}}}, \quad t \geq 0, \\
& \text { (ii) } \lim _{t \rightarrow \infty}\left|\omega_{i}(t)-\omega_{j}(t)\right|=0, \quad 1 \leq i, j \leq N .
\end{aligned}
$$

Proof. The proof for (ii) directly follows from the estimate in (i):

$$
\left|\omega_{i}(t)-\omega_{j}(t)\right| \leq 2 \sqrt{N} \sigma_{\omega}(t) \leq 2 \sqrt{N} \sigma_{\omega}(0) e^{-\frac{\lambda t}{\sqrt{2}}} \rightarrow 0, \quad \text { as } t \rightarrow \infty .
$$

We now consider the estimate (i). Since $\sigma_{\theta}(0)<\frac{\pi}{16 \sqrt{N}}$ and $\sigma_{\theta}$ is a continuous function of $t$, there exists a maximal time $T_{*} \in(0, \infty]$ such that

$$
\sup _{0 \leq t \leq T_{*}} \sigma_{\theta}(t) \leq \frac{\pi}{8 \sqrt{N}}
$$

Step A (Decay estimate in the time zone $\left.\left[0, \mathbf{T}_{*}\right)\right)$ : In this time zone, the condition (3.5) and relation (3.4) through Proposition 3.1 imply

$$
\sigma_{\omega}(t) \leq \sigma_{\omega}(0) e^{-\frac{\lambda t}{\sqrt{2}}}
$$

On the other hand, the first inequality in (3.4) yields

$$
\left|\sigma_{\theta}(t)-\sigma_{\theta}(0)\right| \leq \int_{0}^{t}\left|\frac{d \sigma_{\theta}(s)}{d s}\right| d s \leq \int_{0}^{t} \sigma_{\omega}(s) d s \leq \frac{\sqrt{2}}{\lambda} \sigma_{\omega}(0)<\frac{\pi}{16 \sqrt{N}} .
$$

Hence we have

$$
\sigma_{\theta}(t)<\sigma_{\theta}(0)+\frac{\pi}{16 \sqrt{N}}<\frac{\pi}{8 \sqrt{N}}, \quad t \in\left[0, T_{*}\right] .
$$


Step B $\left(\mathbf{T}_{*}=\infty\right)$ : Suppose the maximal time $T_{*}$ is finite, i.e., $T_{*}<\infty$. Then by the estimate given in Step A, we have

$$
\sigma_{\theta}\left(T_{*}\right)<\frac{\pi}{8 \sqrt{N}}, \quad \sigma_{\omega}\left(T_{*}\right) \leq \sigma_{\omega}(0)<\frac{\lambda \pi}{16 \sqrt{2 N}} .
$$

Hence by the same argument given in Step A, we can find a $\delta>0$ such that

$$
\sup _{0 \leq t \leq T_{*}+\delta} \sigma_{\theta}(t) \leq \frac{\pi}{8 \sqrt{N}} .
$$

This contradicts the maximality of $T_{*}$. Hence $T_{*}=\infty$ and the proof is complete.

Acknowledgment. This research was carried out when S.-Y. Ha was visiting the Department of Mathematics, University of L'Aquila, Italy, and it is a great pleasure to thank the faculty of the applied mathematics group and their hospitality. The work of S.-Y. Ha is partially supported by KRF-2008-C00060 and research grant of SNU-CNS; the work of C. Lattanzio and B. Rubino is partially supported by the Italian MIUR under the PRIN program Nonlinear Systems of Conservation Laws and Fluid Dynamics; the work of M. Slemrod is partially supported by NSF grant DMS-0647554.

\section{REFERENCES}

[1] Acebron, J. A. and Bonilla, L. L.: The Kuramoto model: A simple paradigm for synchronization phenomena. Rev. Mod. Phys. 77, 137-185 (2005).

[2] Artstein, Z. and Vigodner, A.: Singularly perturbed ordinary differential equations with dynamic limits. Proc. Roy. Soc. Edinburgh Sec. A. 126 (1996), 541-569. MR.1396278 (97g:34073)

[3] Carrillo, J. A., Fornasier, M., Rosado, J. and Toscani, G.: Asymptotic flocking dynamics for the kinetic Cucker-Smale model. Preprint.

[4] Cucker, F. and Smale, S.: On the mathematics of emergence. Japan. J. Math. 2, 197-227 (2007). MR 2295620 (2007m:91126)

[5] Cucker, F. and Smale, S.: Emergent behavior in flocks. IEEE Trans. Automat. Control 52, 852-862 (2007). MR2324245(2008h:91132)

[6] Erdmann, U., Ebeling, W. and Mikhailov, A.: Noise-induced transition from translational to rotational motion of swarms. Phys. Review E 71, 051904 (2005).

[7] Ha, S.-Y. and Liu, J.-G.: A simple proof of the Cucker-Smale flocking dynamics and mean-field limit. Commun. Math. Sci. 7, 297-325 (2009). MR2536440

[8] Ha, S.-Y. and Tadmor, E.: From particle to kinetic and hydrodynamic description of flocking. Kinetic and Related Models. 1, 415-435 (2008). MR2425606

[9] Ha, S.-Y. and Slemrod, M.: Flocking dynamics of singularly perturbed oscillator chain and the Cucker-Smale system. J. Dyna. Differential Equations. 21, 371-376 (2009).

[10] Kuramoto, Y.: Chemical oscillations, waves and turbulence. Springer-Verlag, Berlin, 1984. MR762432 (87e:92054)

[11] O'Malley, R. E.: Singular perturbation methods for ordinary differential equations. Applied Mathematical Sciences, 89. Springer-Verlag, New York, 1991. MR.1123483 (92i:34071)

[12] Shen, J.: Cucker-Smale flocking under hierarchical leadership. SIAM J. Appl. Math. 68, 694-719 (2007/08). MR 2375291(2008k:92066)

[13] Tikhonov, A. N.: Systems of differential equations containing a small parameter multiplying the derivative. Mat. Sb. N.S. 27, 147-156 (1950). MR0036902 (12:181d)

[14] Verhulst, F.: Methods and applications of singular perturbations. Boundary layers and multiple timescale dynamics. Texts in Applied Mathematics, 50, Springer, New York, 2005. MR2148856 (2006k:34001) 